\title{
The Fleischner Society 2017 and British Thoracic Society 2015 guidelines for managing pulmonary nodules: keep calm and carry on
}

\author{
Arjun Nair, ${ }_{1}^{1}$ Anand Devaraj, ${ }_{1}^{2}$ Matthew E J Callister, ${ }^{3}$ David R Baldwin ${ }^{4}$
}

\begin{abstract}
- Additional material is published online only. To view please visit the journal online (http://dx.doi.org/10.1136/ thoraxjnl-2018-211764).

${ }^{1}$ Department of Radiology, University College London Hospital, London, UK

${ }^{2}$ Department of Radiology, Royal Brompton Hospital, London, UK ${ }^{3}$ Department of Respiratory Medicine, Leeds Teaching Hospitals NHS Trust, Leeds, UK ${ }^{4}$ Respiratory Medicine Unit, David Evans Research Centre, Nottingham University Hospitals, Nottingham, UK
\end{abstract}

Correspondence to Dr Arjun Nair, Department of Radiology, University College London Hospital, London NW1 2BU, UK; arjun7764@gmail.com

Received 2 March 2018 Revised 19 April 2018 Accepted 14 May 2018 Published Online First 21 June 2018
Check for updates

To cite: Nair A, Devaraj A, Callister MEJ, et al. Thorax 2018:73:806-812.

\section{INTRODUCTION}

Pulmonary nodules are common, with a prevalence of $14 \%-36 \%,{ }^{1-5}$ usually less in case series of nodules that are incidentally detected on thoracic CT scans performed for a variety of clinical indications. Clinical management of CT-detected pulmonary nodules was based initially on a statement made by the Fleischner Society in $2005 .^{6}$ Three further guidelines and one statement have been published since 2013 incorporating emerging evidence. The Fleischner Society provided specific recommendations for subsolid nodule management in 2013. ${ }^{7}$ In the same year, the American College of Chest Physicians (ACCP) issued nodule guidance as part of its recommendations for the diagnosis and management of lung cancer. ${ }^{8}$ In 2015, the British Thoracic Society (BTS) published guidelines for nodule management, accompanied by a comprehensive evidence review. ${ }^{9}$ Most recently, the Fleischner Society updated their guidance for management and measurement of nodules, replacing both their 2005 and 2013 guidance. ${ }^{1011}$ Clinician awareness of, and adherence to, such guidance has historically been poor, and remains so. ${ }^{12-16}$ Guidelines that differ in their recommendations are a potential source of confusion and thus risk increasing variation in clinical practice rather than achieving one of the aims of guidelines: improving uniformity of clinical management.

In this paper, we compare the two most recent guidelines, the BTS 2015 and Fleischner 2017 guidelines, describing the many helpful similarities and elaborating on the areas where there is divergence in recommendations. We also provide a more indepth discussion in the online supplementary material.

The respective approaches to solid and subsolid nodules (SSNs) are summarised in table 1.

\section{SIMILARITIES AND AREAS OF AGREEMENT}

The BTS and Fleischner guidelines, like others, deal with the issue of distinguishing the minority of pulmonary nodules that are malignant from the majority that are benign. Both guidelines draw heavily from the extensive evidence base provided by CT lung cancer screening trials over the last two decades.

Nodules with unequivocal benign features such as the presence of popcorn calcification require no further follow-up in both guidelines. Furthermore, both guidelines recommend that the so-called perifissural nodules (PFNs), with typical polygonal, even flat or ovoid morphology, do not require

\section{Key messages}

What is the key question?

- How do the Fleischner recommendations for the management of incidental pulmonary nodules published in 2017 compare with the British Thoracic Society (BTS) recommendations published in 2015?

What is the bottom line?

- The updated Fleischner Society recommendations are mostly similar to the 2015 BTS guideline, but the BTS goes further in its recommendations concerning the use of risk prediction models and volumetry, while the Fleischner guidelines go further in recommending a conservative approach to stable pure ground-glass nodules.

Why read on?

- We discuss the areas where the two guidelines diverge - namely routes of presentation, solid nodule management and risk assessmentwith reference to their rationale, relative merits and drawbacks, and offer pragmatic conclusions relevant to clinical practice about which guideline to adopt.

follow-up, as these are often benign and mostly represent intrapulmonary lymph nodes (IPLNs). ${ }^{17-19}$ However, agreement on what a 'typical' PFN is can be difficult (figure 1), and any concerning features such as irregular margins or pleural distortion should prompt a follow-up CT.

Recommendations for nodules without unequivocal benign features are first stratified by the composition of a nodule (solid, part-solid or ground-glass), followed by nodule size and an assessment of risk of malignancy, incorporating nodule and patient factors, where appropriate. This assessment of risk in both sets of guidelines includes what are regarded as the most dominant risk factors, namely age and tobacco smoking history.

Both guidelines emphasise the need for low-radiation dose, thin-section contiguous CT and consistency in technical factors between examinations as far as possible; the BTS and Fleischner recommendations are for maximum $\mathrm{CT}$ reconstruction thicknesses of $1.25 \mathrm{~mm}$ and $1.5 \mathrm{~mm}$, respectively. 


\section{DIFFERENCES}

\section{Scope}

The 2017 Fleischner guidelines primarily cover the radiological management and measurement of incidentally detected pulmonary nodules, in contrast to the BTS, which deals comprehensively with all aspects of nodule management (see online supplementary box 1 for topics covered by each guideline).

\section{Target population and route of presentation}

The Fleischner guidelines are only applicable to patients aged at least 35 years in whom there is no prior diagnosis of cancer, and do not pertain to lung cancer screening-detected nodules. ${ }^{11}$ In contrast, the guideline development group of the BTS found insufficient evidence to recommend different approaches to managing pulmonary nodules according to their route of presentation, so the guidelines can be applied to adults aged 18 or over with pulmonary nodules irrespective of the route of detection. ${ }^{9}$ The BTS guidelines acknowledge that there is conflicting comparative evidence with respect to nodules in patients with previous malignancy and adopt a cautious approach. They recommend that the presence of previous malignancy is considered as a factor in the baseline assessment of nodules and in the follow-up of larger IPLNs ( $\geq 10 \mathrm{~mm}$ ).

These differences should be appreciated in the wider context of guideline development. In the USA, guidance on the investigation and management of pulmonary nodules, including further surgical and non-surgical management, is already available from the ACCP. ${ }^{8}$ Lung cancer screening is currently recommended in the USA, ${ }^{20}$ and programmes are required to adhere to a more protocol-driven (but also diameter-based), quality-assured standard of reporting such as the American College of Radiology Lung CT Screening Reporting and Data System (Lung-RADS). ${ }^{21}$ In contrast, lung cancer screening is not yet recommended or widely available in the UK. As such, the terms of reference of the Fleischner guidelines could be considered deliberately and appropriately more restrictive.

\section{Solid nodules}

\section{Determination of nodule size}

The BTS guideline was the first to make a recommendation for a radical departure from conventional assessment of baseline size and subsequent growth by recommending semiautomated volumetry, where available and reliable, as the new standard. The rationale for this change is discussed in the online supplementary material, but was primarily based on convincing high-quality evidence from the Dutch-Belgian Randomized Lung Cancer Screening Trial (Nederlands-Leuvens Longkanker Screenings Onderzoek (NELSON)). ${ }^{22}$ The maximum, not average, diameter to determine nodule size is recommended only when accurate volumetry is not achievable. ${ }^{9}$

In contrast and in accord with all other extant guidelines, the Fleischner 2017 guidelines continue to recommend diameter measurements to determine nodule size, and have produced a companion paper as a further guide. ${ }^{10}$ The stipulation is to use the plane (transverse, sagittal or coronal) which illustrates the nodule in its two greatest dimensions and take the average of the long-axis and perpendicular short-axis diameters in that plane used to define nodule size (manipulation of images into non-orthogonal orientations is not recommended). Software-derived volumetric assessment is acknowledged as an alternative measurement tool but is not recommended as the standard of care. However, alternative thresholds for follow-up based on volume are defined (see below), with the caveat that identical software versions be used on baseline and follow-up scans when assessing growth using volumetry. ${ }^{10}$

The Fleischner guidelines also recommend rounding nodule diameter to the nearest whole millimetre, rather than increments of $0.1 \mathrm{~mm}$, as such fractional millimetre measurements are thought to spuriously convey accuracy when they are in fact imprecise (see online supplementary material). Consequently, a nodule measuring $5.5 \mathrm{~mm}$ would be considered $6 \mathrm{~mm}$, while a measurement of $5.4 \mathrm{~mm}$ would be considered $5 \mathrm{~mm}$. For spiculated nodules, only the solid 'core' of the nodule should be measured, omitting the spiculations. Nodules perceived to be $3 \mathrm{~mm}$ or smaller can be termed micronodules ${ }^{23}$ and require no formal measurement. ${ }^{10}$

\section{Size thresholds for follow-up}

Both guidelines are conceptually similar in that they (1) raise the size thresholds for follow-up compared with earlier recommendations (please also see online supplementary material); and (2) divide solid nodules into small, medium and large categories (although these categories are not explicitly labelled as such) where no action, imaging follow-up and more aggressive investigation are recommended, respectively (table 1 ).

According to the Fleischner guidelines, these categories are defined using average diameters rounded to $<6 \mathrm{~mm}, 6-8 \mathrm{~mm}$ and $>8 \mathrm{~mm}$, respectively, while the corresponding BTS definitions are maximum diameters of $<5 \mathrm{~mm}, 5-8 \mathrm{~mm}$ or $>8 \mathrm{~mm}$, respectively. The volume-based Fleischner thresholds for the so-called small, medium and large nodules are $<100 \mathrm{~mm}^{3}$ (roughly equivalent to the volume of a sub- $6 \mathrm{~mm}$ diameter sphere), $100-250 \mathrm{~mm}^{3}$ and $>250 \mathrm{~mm}^{3}$ (roughly equivalent to the volume of a $>8 \mathrm{~mm}$ diameter sphere), respectively. The corresponding volume thresholds in the BTS guidelines are $<80 \mathrm{~mm}^{3}$, $80-300 \mathrm{~mm}^{3}$ and $\geq 300 \mathrm{~mm}^{3}$, respectively. The BTS guideline lowered the volume cut-off to $80 \mathrm{~mm}^{3}$ from $100 \mathrm{~mm}^{3}$ to take into account interscan and intersoftware variation, since the $100 \mathrm{~mm}^{3}$ threshold is derived from only a single type of volumetric software used in the NELSON study.

The Fleischner guidelines retain the option of a further follow-up at 12 months for solid nodules $<6 \mathrm{~mm}$ or $<100 \mathrm{~mm}^{3}$ when there are patient or nodule risk factors that are associated with a higher pretest probability of malignancy (approximating to $>1 \%$ ), such as upper lobe location or nodule morphology; however, spiculation is the only morphological feature relevant to solid nodules that is discussed. The BTS guidelines, drawing on evidence that diameter measurement of smaller nodules cannot reliably detect growth at a 3-month interval, recommend a 12-month follow-up for those with a maximum diameter of $5-6 \mathrm{~mm}$, where volumetric assessment is not possible.

\section{Follow-up intervals and total duration}

The Fleischner guidelines recommend a range of time intervals for follow-up depending on nodule size, as opposed to the specific time intervals prescribed by the BTS. The Fleischner recommendation that solid nodules $6-8 \mathrm{~mm}$ be followed with a CT scan in 6-12 months in the first instance, regardless of risk, is later than the 3 months advocated by the BTS for similar-sized nodules based on evidence from the Dutch-Belgian Randomized Lung Cancer Screening Trial (NELSON) ${ }^{22}$ and the pilot UK Lung Cancer Screening ${ }^{24}$ trial. The Fleischner authors acknowledge that earlier follow-up than stipulated may be required if a patient is anxious.

Provided nodules show no significant growth on initial follow-up CT, both guidelines recommend a total of 24 months 
Table 1 Key differences between the Fleischner Society 2017 and the British Thoracic Society 2015 recommendations for pulmonary nodules

\begin{tabular}{|c|c|c|}
\hline & Fleischner 2017 & British Thoracic Society guideline 2015 \\
\hline Scope & Primarily radiological management. & Comprehensive management, from imaging to intervention. \\
\hline Applicability & Incidentally detected pulmonary nodules. & All pulmonary nodules regardless of presentation route. \\
\hline Target population & Age 35 or older. & Age 18 or older. \\
\hline \multicolumn{3}{|l|}{ Solid nodules } \\
\hline $\begin{array}{l}\text { Recommended sizing } \\
\text { method* }^{*}\end{array}$ & $\begin{array}{l}\text { Average short-axis and long-axis diameter (in the orthogonal plane } \\
\text { which gives the largest two measurements). } \\
\text { Diameter rounded to the nearest whole millimetre. } \\
\text { (Volumetry parameters are also specified.) }\end{array}$ & $\begin{array}{l}\text { Volumetry. } \\
\text { Maximum diameter if volumetry unreliable/ unavailable. }\end{array}$ \\
\hline $\begin{array}{l}\text { Size threshold for } \\
\text { follow-up }\end{array}$ & $\begin{array}{l}6 \mathrm{~mm} \text { (NB: } 5.5 \mathrm{~mm} \text { or greater would be rounded up to } 6 \mathrm{~mm} \text { ). } \\
\text { Caveat: Certain patients at high risk with suspicious nodule } \\
\text { morphology, upper lobe location or both may warrant 12-month follow- } \\
\text { up. }\end{array}$ & $\begin{array}{l}80 \mathrm{~mm}^{3} \text { or } 5 \mathrm{~mm} \text {. } \\
\text { Caveat: Nodules smaller than this threshold which are definitely new compared with CT } \\
\text { performed within the last } 1-2 \text { years merit follow-up. }\end{array}$ \\
\hline $\begin{array}{l}\text { Definition of } \\
\text { significant growth* }\end{array}$ & $\geq 2 \mathrm{~mm}$ diameter. & $\begin{array}{l}>25 \% \text { increase in volume (NB: use diameter measurements where volumetry is not possible } \\
\text { or where there is clear evidence of marked growth). }\end{array}$ \\
\hline Follow-up intervals & $\begin{array}{l}\text { Range of intervals rather than specified interval. } \\
\text { Medium ( } 6-8 \mathrm{~mm}, 100-250 \mathrm{~mm}^{3} \text { ) nodules: } \\
\text { Solitary: CT at } 6-12 \text { months, then CT at } 18-24 \text { months (consider need } \\
\text { for longer follow-up if high risk). } \\
\text { Multiple: CT at } 3-6 \text { months, then consider CT at } 18-24 \text { months; use } \\
\text { largest nodule to guide management. } \\
\text { Larger ( }>8 \mathrm{~mm},>250 \mathrm{~mm}^{3} \text { ) nodules: consider CT at } 3 \text { months, PET/CT or } \\
\text { tissue sampling. }\end{array}$ & $\begin{array}{l}\text { Prespecified intervals }(3,12 \text { and } 24 \text { months). } \\
\left.\text { Medium ( } 5-8 \mathrm{~mm}, 80-300 \mathrm{~mm}^{3}\right) \text { nodules: } \\
\mathrm{CT} \text { at } 3 \text { months; further follow-up based on VDT. } \\
\text { If diameter used, } 5-6 \mathrm{~mm} \text { nodules to be followed at } 12 \text { months. } \\
\text { No separate recommendation for multiple nodules; Brock model incorporates nodule count. } \\
\text { Use largest nodule to guide management. } \\
\left.\text { Larger ( }>8 \mathrm{~mm},>300 \mathrm{~mm}^{3}\right) \text { nodules: } \\
\text { Perform risk estimation with Brock model: risk } \geq 10 \% \text { merits PET-CT; subsequent follow-up } \\
\text { dictated by Herder model risk assessment. } \\
\text { (Herder score: }<10 \% \text { CT surveillance; } 10 \%-70 \% \text { - consider image-guided biopsy, excision } \\
\text { or surveillance; }>70 \% \text { consider definitive treatment.) }\end{array}$ \\
\hline $\begin{array}{l}\text { Follow-up } \\
\text { duration (assuming } \\
\text { interscan stability) }\end{array}$ & $\begin{array}{l}\text { Well-defined solid nodules with benign morphology at } 12-18 \text { months } \\
\text { can be discontinued if the nodule is 'accurately measurable and } \\
\text { unequivocally stable'. }\end{array}$ & $\begin{array}{l}\text { If volumetry reliably used: } \\
\text { If volume stable ( }<25 \% \text { change), discharge at } 12 \text { months; if VDT }>600 \text { days, consider } \\
\text { discharge but option for CT surveillance. } \\
\text { If diameter used: } \\
24 \text { month follow-up. }\end{array}$ \\
\hline
\end{tabular}

Subsolid nodules

Size threshold for

follow-up

.

Follow-up intervals

Follow-up duration (assuming interscan stability)

Risk assessment*
Single: $6 \mathrm{~mm}$ (NB: $5.5 \mathrm{~mm}$ or greater would be rounded up to $6 \mathrm{~mm}$ ). Caveat: In certain suspicious nodules $<6 \mathrm{~mm}$, consider follow-up at 2 and 4 years (eg, risk factors such as Asian populations). If solid component(s) or growth develops, consider resection. Multiple: no lower threshold.

Small $(<6 \mathrm{~mm})$ single and multiple:

$\mathrm{CT}$ at 3-6 months.

If stable, consider CT at 2 and 4 years. Common recommendation for both pure ground-glass and part-solid nodules.

Larger $\left(\geq 6 \mathrm{~mm}, \geq 100 \mathrm{~mm}^{3}\right)$

- Single ground-glass: CT at 6-12 months to confirm persistence, then CT every 2 years.

- Single part-solid: CT at 3-6 months to confirm persistence. If unchanged and solid component remains, $6 \mathrm{~mm}$, perform annual CT.

- Multiple (ground-glass or part-solid): CT at 3-6 months. Subsequent management based on the most suspicious nodule(s).

- Consider resection for growing nodules or those that develop a solid component.

Same recommendation for pure ground-glass and part-solid nodules.

Single: 5 years.

Multiple: dictated by the largest nodule.

Applied to all nodule sizes.

Modified ACCP guidance.

$\rightarrow$ Low risk: $<5 \%$.

High risk: $\geq 5 \%$.

Assignment of risk primarily based on known risk factors (age, smoking Herder model following PET-CT.

history, family history, nodule morphology, spiculation, upper lobe

location, emphysema, pulmonary fibrosis).

No specific model-based quantification suggested.
$5 \mathrm{~mm}$.

Caveat: no follow-up even if $\geq 5 \mathrm{~mm}$ if patient unfit for any treatment or stability from previous $C T$ over 4 years.

No separate recommendation for single or multiple nodules.

Small ( $<5 \mathrm{~mm})$ : no follow-up.

Larger $(\geq 5 \mathrm{~mm})$ : repeat $\mathrm{CT}$ in 3 months.

- Stable: assess risk of malignancy with Brock model and morphology, patient fitness and patient preference.

Caveat: Brock model may underestimate malignancy risk in persistent subsolid nodules.

- Low risk (approximate Brock estimate $<10 \%$ ): CT at 1, 2 and 4 years from baseline.

- High risk (approximate Brock estimate $>10 \%$ ) or concerning morphology, for example, solid component presence or growth, pleural indentation, vacuolation: discuss options for CT surveillance, image-guided biopsy, resection or non-surgical treatment.

- Growth: resection, non-surgical treatment or observation (see main text for discussion).

- Altered morphology (increasing density/new solid component): favour resection/nonsurgical treatment.

4 years

For solid nodules, risk assessment only suggested for large $\left(>8 \mathrm{~mm},>300 \mathrm{~mm}^{3}\right)$ nodules. Brock model.

Low risk: $<10 \%$

High risk: $\geq 10 \%$

- Low risk: $<10 \%$

- Intermediate risk: $\geq 10 \%-70 \%$

- High risk: $>70 \%$

*Denotes parameters where the guidelines differ, which are potentially most likely to create the greatest divergence in management recommendations. It should be noted that this is solely the view of the authors, in the absence of evidence comparing the two guidelines.

ACCP, American College of Chest Physicians; PET, positron emission tomography; VDT, volume doubling time.

of follow-up to assess stability when nodules have been assessed using diameter. However, patients with nodules that have been stable or demonstrated no significant growth (see below) with reliable volumetry can be discharged at 12 months according to the BTS guidelines, while the Fleischner view is that follow-up of well-defined solid nodules with benign morphology at 12-18 

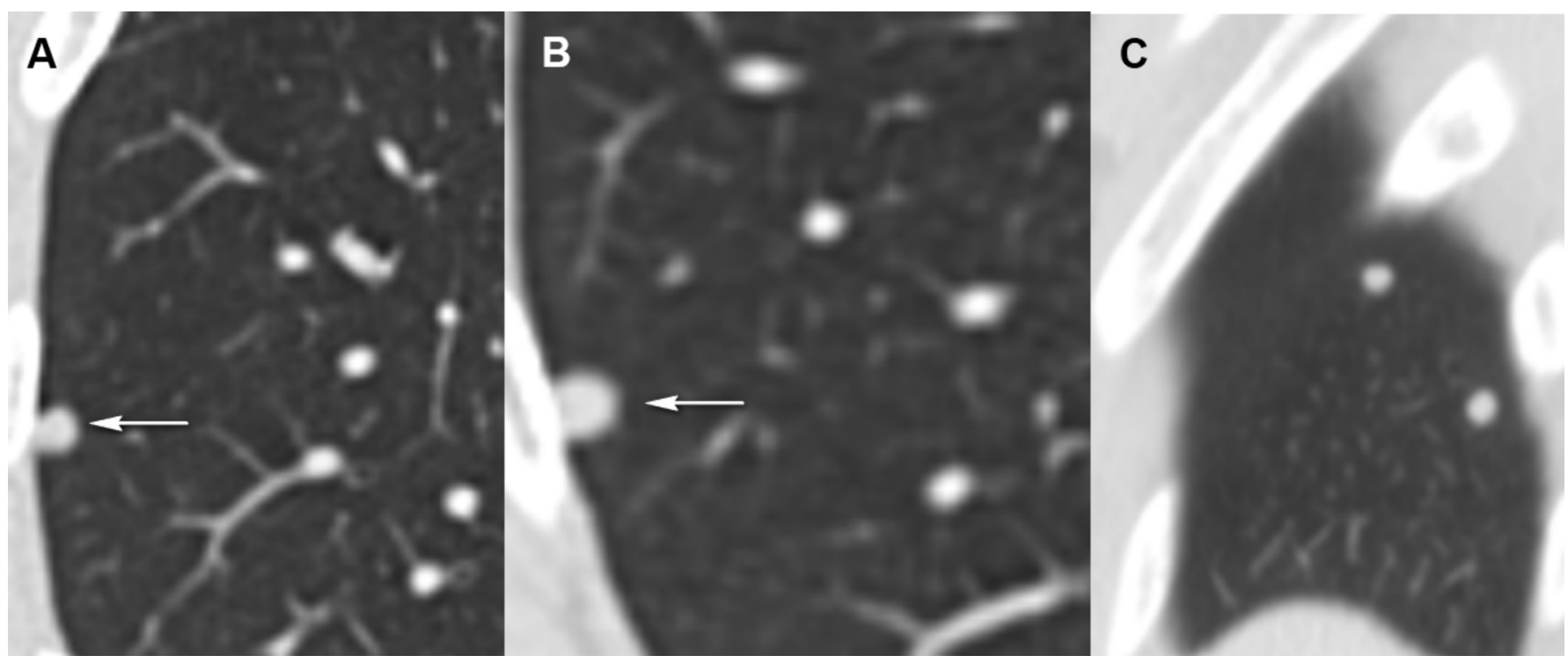

Figure 1 Two $6 \mathrm{~mm}$ and $7 \mathrm{~mm}$ right lower lobe subpleural nodules (white arrows in A and B) which are round rather than polygonal in a 50 -yearold non-smoker. Sagittal images are often useful in confirming whether the nodules are in fact 'flat' (reassuring for an IPLN), but in this case the nodules appear round on sagittal reconstructions as well (C). Both the Fleischner and the British Thoracic Society guidelines advocate no further follow-up for typical PFNs/IPLNs; however, in this case two observers considered the nodules atypical and advocated follow-up, while a third thoracic radiologist thought these were typical. Such variation can lead to heterogeneous application of either guideline. IPLN, intrapulmonary lymph nodes; PFN, perifissural nodules.

months can be discontinued if the nodule is 'accurately measurable and unequivocally stable', although a definition of this term is not provided. ${ }^{11}$

\section{Definition of significant growth}

The Fleischner guidelines recommend that a nodule (both solid and subsolid) can only reliably be said to have grown when it has increased by at least $2 \mathrm{~mm}$ in diameter, given that the upper limit of interobserver variation in diameter measurement is around $1.73 \mathrm{~mm} .{ }^{25}$ In contrast, the prescription of volume doubling time (VDT) measurement in the BTS guidelines allows for nodules that may be growing more subtly at first follow-up to be detected earlier (figure 2), while volumetric stability (allowing for stability of technical and software factors) allows earlier discharge and so earlier reassurance. We discuss the implications of these different definitions of growth in detail in the 'Volumetric Analysis' section in the online supplementary material. The BTS guidelines define a solid nodule as growing only if its volume has increased by $>25 \%$ as this is the magnitude of interscan variation that can occur in volumetric measurement. ${ }^{26}$ Provided a $>25 \%$ increase is seen, the BTS guidelines then further risk-stratify solid nodules that are not definitely stable on diameter or volume: a VDT of $\leq 400$ days is considered suspicious for malignancy for which definitive management is advocated at all time-points (3, 12 and, if relevant, 24 months). It is worth noting that in practice, at 3 months, a nodule which had increased by $>25 \%$ would have a VDT of less than 280 days in any case. Nodules with VDT $>400$ and $\leq 600$ days require an informed discussion with a patient as to the options available, while conservative management is recommended for nodules with VDT > 600 days. VDTs can also be calculated on the basis of manual diameter measurements, although the evidence for this was limited to nodules measured manually in NELSON, from which VDT was calculated.

\section{Intervention strategies for larger nodules}

For larger nodules $\left(>8 \mathrm{~mm},>250 \mathrm{~mm}^{3}\right)$, the Fleischner guidelines suggest one or a combination of three options: 3 -month follow-up, positron emission tomography (PET)-CT or tissue sampling, in both low-risk and high-risk patients, with no particular guidance on how this choice could ultimately be made. The BTS uses pretest probability to guide the decision to proceed to PET-CT, followed by post-test probability from PET-CT, to guide the decision for surveillance, sampling or definitive management. The latter was guided by an evidence review from which the negative likelihood ratio of transthoracic needle biopsy was derived.

\section{Risk assessment}

The Fleischner guidelines adapt the ACCP 2013 guidance for risk. The ACCP recommends that in the individual with a solid, indeterminate nodule that measures $>8 \mathrm{~mm}$ in diameter, the clinician should 'estimate the pre-test probability of malignancy either qualitatively by using their clinical judgment and/or quantitatively by using a validated model'. 8 The validated models quoted in the ACCP guidance include those published by the Veterans Association, Mayo Clinic and Prostate, Lung, Colorectal and Ovarian (PLCO) Cancer Screening Trial (2009). ${ }^{27-29}$ The Fleischner guidelines modify the interpretation of this ACCP assessment of risk in three ways. First, they extrapolate the ACCP guidance to nodules of all sizes. Next, they dichotomise the ACCP categories of low $(<5 \%)$, intermediate $(5 \%-65 \%)$ and high $(>65 \%)$ risk to low $(<5 \%)$ and high $(\geq 5 \%)$ risk categories only. Finally, although such categories are explicitly defined, the Fleischner guidance implies that assignment of these risk categories is primarily based on an impressionistic synthesis of known risk factors, namely age, smoking history, family history, nodule morphology (spiculation in particular), upper lobe location, and also emphysema and pulmonary fibrosis, rather than modelbased multifactorial quantification with prespecified thresholds. 


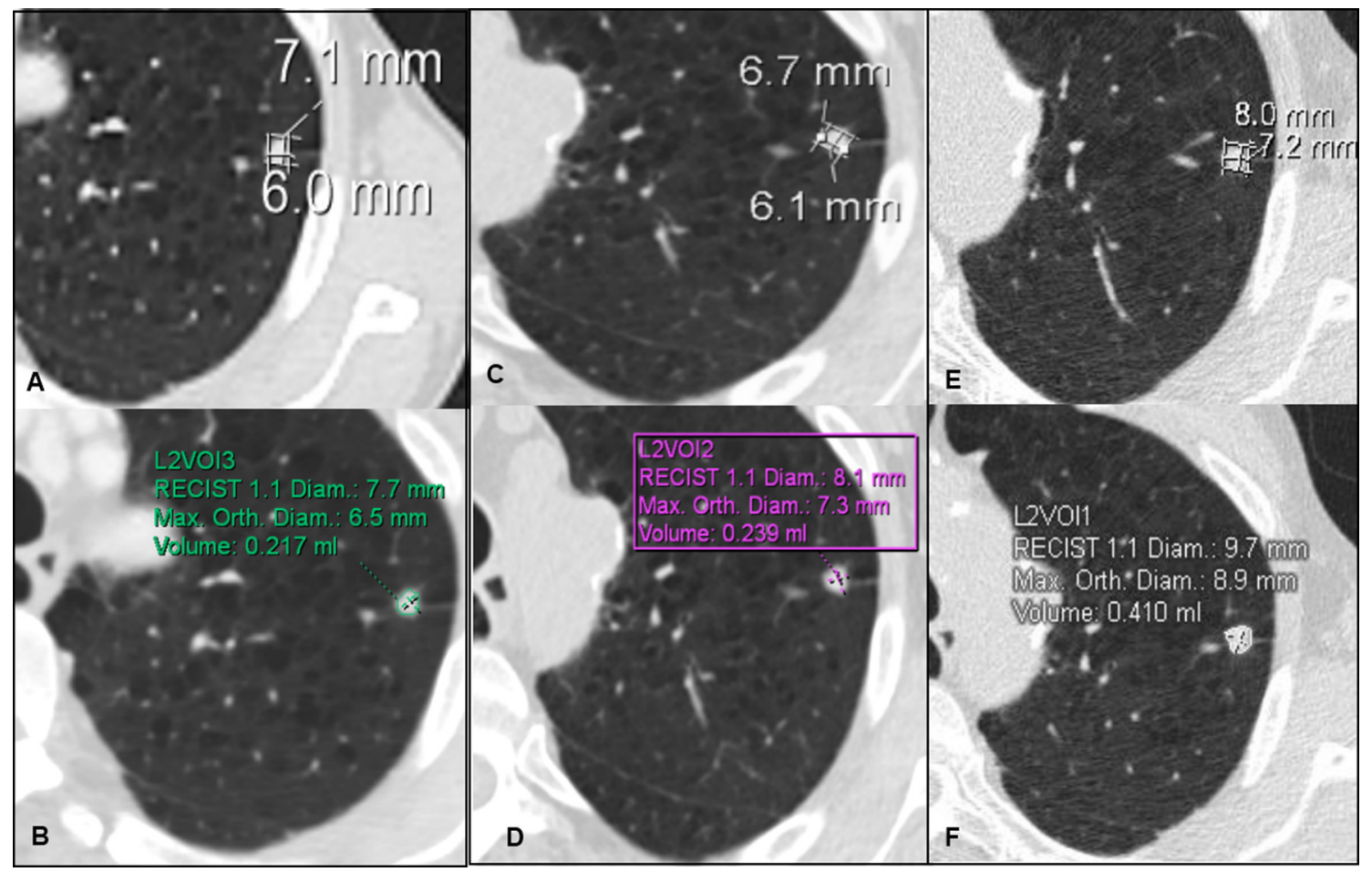

Figure 2 Baseline ( $A$ and $B), 3$-month ( $C$ and $D)$ and 12-month ( $E$ and $F$ ) manual diameter and volume measurements of a left upper lobe nodule showing diameter stability (1 mm average diameter change over 12 months) and initial volumetric stability, but longer term volumetric growth, with a volume doubling time of 350 days over 12 months. The patient's cardiac comorbidities and COPD precluded a histological diagnosis or surgical resection, and the patient was given the option of stereotactic ablative radiotherapy.

This is particularly relevant when considering follow-up of sub-6 mm nodules and could place a significant burden on clinical teams in estimating risk and conveying this to patients.

The BTS, on the other hand, uses the multifactorial Brock model to assess pretest probability, derived and validated from two separate Canadian lung cancer screening and chemoprevention study cohorts (the PanCan and British Columbia Cancer Agency data sets). ${ }^{30}$ This risk assessment is only invoked for nodules $\geq 300 \mathrm{~mm}^{3}$ or $\geq 8 \mathrm{~mm}$ because smaller nodules rarely exceed the risk threshold used to define high risk $(\geq 10 \%)$, where PET-CT is subsequently recommended. Post-test probability of malignancy following a PET-CT is assessed using the Herder model, derived from 106 patients with incidental pulmonary nodules ${ }^{31}$ and subsequently externally validated. In combination, these models incorporate all the known risk factors recommended by the Fleischner guidelines, apart from pulmonary fibrosis. It is useful to bear in mind that, as the Brock model was developed and validated in cohorts of ever-smokers, smoking status and smoking index (pack-years) did not discriminate between benign and malignant nodules, ${ }^{30}$ and are thus not included in the model. Conversely, smoking status is included in the Herder model; as such, in the BTS guideline, smoking status is only incorporated into risk assessment for nodules $\geq 300 \mathrm{~mm}^{3}$ or $\geq 8 \mathrm{~mm}$ after PET-CT has been performed.

Using different models can lead to a variation in calculated risk (figure 3). The implications of the different risk assessment approaches by the two guidelines are further explored in the online supplementary material.

\section{Special scenarios}

\section{Multiple nodules}

In the case of multiple solid nodules, both the Fleischner and BTS guidelines recommend using the largest nodule to guide management. The Fleischner guidelines make specific recommendations for multiple nodules, and in particular advocate shorter intervals (3-6 months in the first instance) for medium-sized solid nodules, regardless of risk (table 1).

New nodules on follow-up CT scans

The Fleischner guidelines make no specific management recommendation for new nodules discovered on follow-up CT scans. The published BTS guideline likewise acknowledged a dearth of evidence regarding the management of such nodules and made no specific recommendations for their management. However, since the publication of the BTS guideline, strong evidence from the NELSON trial has emerged, indicating that smaller nodules (with volumes $>27 \mathrm{~mm}^{3}$ ) on incident screening rounds have an at least intermediate probability $(>3 \%)$ of lung cancer. ${ }^{32}$ Thus, the subsequent BTS Pulmonary Nodules Quality Standards and the BTS mobile platform application, developed in partnership with Cancer Research UK, ${ }^{33}$ now include a recommendation to consider follow-up for nodules, even if small, which are definitely new compared with a CT from 1 to 2 years previously.

\section{Subsolid nodules}

Both guidelines recommend a conservative approach to pure ground-glass nodules (pGGNs), with a more aggressive approach for growing pGGNs and part-solid nodules (PSNs) with larger solid components. In doing so, they both acknowledge the more indolent nature of SSNs-the umbrella term for both these nodule types, especially pGGNs which, by definition, lack a solid component.

Imaging follow-up is favoured for $\mathrm{pGGN} \geq 5 \mathrm{~mm}$ in maximum diameter (according to BTS) or $\geq 6 \mathrm{~mm}$ in average diameter (according to Fleischner guidelines). Follow-up duration is 


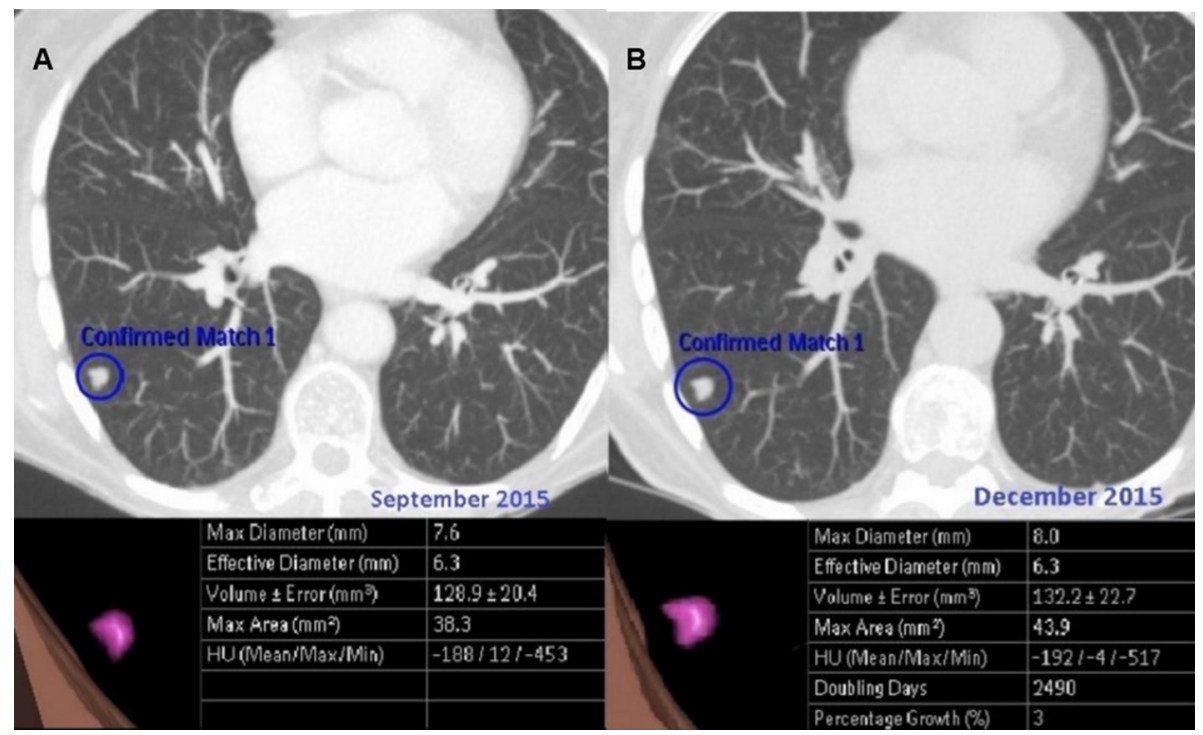

Figure 3 A right lower lobe nodule measures $8 \mathrm{~mm}$ in maximum diameter and $6 \mathrm{~mm}$ in average diameter. Its baseline reliable volume is $129 \mathrm{~mm}^{3}$ (A). According to the British Thoracic Society, the patient would have a repeat CT at 3 months, and then again at 12 months if stable. The nodule was stable at 3 months (B) and at 12 months with a reassuring percentage volume change of $3 \%$, within interscan variation (upper limit of $25 \%$ ). According to the Fleischner guidelines, however, the patient's risk score would first need to be calculated. The Brock score is $5.4 \%$, which would be considered low risk, while the Mayo score is $14.1 \%$, placing the patient in the high-risk category, for which follow-up for 24 months is recommended.

1 year less in the BTS guideline (4 years) than in the Fleischner guideline (5 years). The BTS recommendation for a repeat CT at 3 months for all SSNs $\geq 5 \mathrm{~mm}$, and the Fleischner recommendation for a repeat CT at 6 months regardless of size, reflect the fact that $25 \%$ or more of these nodules are transient inflammatory lesions.

For persistent and stable PSNs as well as pGGNs, the BTS guidelines recommend assessment of malignant risk using the Brock score, with further management guided by that assessment of risk. For those with a Brock score $>10 \%$, surveillance, biopsy, resection or non-surgical treatment are all recommended as considerations based on the patient's age and comorbidities. By contrast the Fleischner guidelines are more prescriptive in that only a conservative observational approach for both pGGN and PSNs with a solid component $<6 \mathrm{~mm}$ is recommended, regardless of risk.

However, both the BTS and Fleischner guidelines recommend a more proactive approach to persistent, solitary PSNs with suspicious features. In the Fleischner guidelines these are SSNs with lobulated margins or cystic components, a growing solid component, or a solid component larger than $8 \mathrm{~mm}$. In the BTS guidelines, suspicious morphology descriptors include SSNs with a large solid component, bubble-like appearance, causing pleural indentation or demonstrating new solid components. Emerging evidence, suggesting a conservative approach could be adopted by both guidelines for the majority of SSNs, is considered in the online supplementary material.

\section{CONCLUSION}

The updated Fleischner Society recommendations are mostly similar to the 2015 BTS guideline. It is interesting to note that, even though published 2 years earlier, the BTS goes further in its recommendations concerning the use of risk prediction models and volumetry. The BTS guidelines place a greater emphasis on evidence-based recommendations such as the use of fixed (rather than a range of) intervals between follow-up CTs, thus eliminating vagueness and variability. However, the Fleischner guidelines go further in recommending a conservative approach to stable pGGN, which may, in future iterations of guidelines, extend to stable PSNs should further data on long-term follow-up become available. Ongoing efforts to understand the magnitude of, and factors influencing, interobserver variation for the more subjective elements of nodule risk prediction models and management algorithms (especially nodule composition, spiculation and metabolic avidity on PET-CT), as well as more detailed investigations into differences between volumetry packages and their potential impact on management, will also likely inform guideline development in the future. However, until then, the BTS guideline remains reassuringly contemporary-so keep calm (with respect to pGGN) and carry on.

Correction notice This article has been corrected since it was published Online First. The second author's name was corrected.

Contributors All authors have made substantial contributions to all of the following: (1) the conception and design of the study, or acquisition of data, or analysis and interpretation of data; (2) drafting the article or revising it critically for important intellectual content; and (3) final approval of the version to be submitted, as per the ICMJE requirements.

Competing interests AN declares that he is a member of the Advisory Board for Aidence BV, The Netherlands, and he is part-funded by the UCLH Biomedical Research Centre (BRC), National Institute. DRB declares grants from AstraZeneca and Oncimmune, outside the submitted work. DRB and MEJC were coauthors of the British Thoracic Society guidelines for the investigation and management of pulmonary nodules published in 2015. AD has no competing interests to declare.

Patient consent Not required.

Provenance and peer review Not commissioned; externally peer reviewed.

(c) Article author(s) (or their employer(s) unless otherwise stated in the text of the article) 2018. All rights reserved. No commercial use is permitted unless otherwise expressly granted.

\section{REFERENCES}

1 Foley PW, Hamaad A, El-Gendi $\mathrm{H}$, et al. Incidental cardiac findings on computed tomography imaging of the thorax. BMC Res Notes 2010;3:326.

2 Henschke Cl, McCauley DI, Yankelevitz DF, et al. Early Lung Cancer Action Project: overall design and findings from baseline screening. Lancet 1999;354:99-105 
3 Diederich S, Wormanns D, Semik M, et al. Screening for early lung cancer with low-dose spiral CT: prevalence in 817 asymptomatic smokers. Radiology 2002;222:773-81

4 Bendix K, Jensen JM, Poulsen S, et al. Coronary dual source multi detector computed tomography in patients suspected of coronary artery disease: prevalence of incidental extra-cardiac findings. Eur J Radiol 2011;80:109-14.

5 Chong S, Lee KS, Chung MJ, et al. Lung cancer screening with low-dose helical CT in Korea: experiences at the Samsung Medical Center. J Korean Med Sci 2005;20:402-8.

6 MacMahon H, Austin JH, Gamsu G, et al. Guidelines for management of small pulmonary nodules detected on CT scans: a statement from the Fleischner Society. Radiology 2005;237:395-400.

7 Naidich DP, Bankier AA, MacMahon H, et al. Recommendations for the management of subsolid pulmonary nodules detected at CT: a statement from the Fleischner Society. Radiology 2013;266:304-17.

8 Gould MK, Donington J, Lynch WR, et al. Evaluation of individuals with pulmonary nodules: when is it lung cancer? Diagnosis and management of lung cancer, 3rd ed: American College of Chest Physicians evidence-based clinical practice guidelines. Chest 2013;143:e93S-120.

9 Callister ME, Baldwin DR, Akram AR, et al. British Thoracic Society guidelines for the investigation and management of pulmonary nodules. Thorax 2015;70(Suppl 2):ii1-ii54.

10 Bankier AA, MacMahon H, Goo JM, et al. Recommendations for Measuring Pulmonary Nodules at CT: A Statement from the Fleischner Society. Radiology 2017; 285:162894-

11 MacMahon H, Naidich DP, Goo JM, et al. Guidelines for Management of Incidental Pulmonary Nodules Detected on CT Images: From the Fleischner Society 2017. Radiology 2017;284:228-43.

12 Lacson R, Prevedello LM, Andriole KP, et al. Factors associated with radiologists' adherence to Fleischner Society guidelines for management of pulmonary nodules. J Am Coll Radiol 2012;9:468-73.

13 Tanner NT, Aggarwal J, Gould MK, et al. Management of Pulmonary Nodules by Community Pulmonologists: A Multicenter Observational Study. Chest 2015;148:1405-14.

14 Mets OM, de Jong PA, Chung K, et al. Fleischner recommendations for the management of subsolid pulmonary nodules: high awareness but limited conformance - a survey study. Eur Radiol 2016;26:3840-9.

15 Tanner NT, Porter A, Gould MK, et al. Physician Assessment of Pretest Probability of Malignancy and Adherence With Guidelines for Pulmonary Nodule Evaluation. Chest 2017:152:263-70.

16 Baldwin DR, Callister MEJ. Physician Assessment of Pretest Probability of Malignancy and Adherence to Guidelines for Pulmonary Nodule Evaluation. Chest 2017;152:447-8.

17 de Hoop B, van Ginneken B, Gietema $\mathrm{H}$, et al. Pulmonary perifissural nodules on $\mathrm{CT}$ scans: rapid growth is not a predictor of malignancy. Radiology 2012;265:611-6.
18 Ahn MI, Gleeson TG, Chan IH, et al. Perifissural nodules seen at CT screening for lung cancer. Radiology 2010;254:949-56.

19 Bankoff MS, McEniff NJ, Bhadelia RA, et al. Prevalence of pathologically proven intrapulmonary lymph nodes and their appearance on CT. AJR Am J Roentgenol 1996;167:629-30

20 Moyer VA; U.S. Preventive Services Task Force. Screening for lung cancer: U.S. Preventive Services Task Force recommendation statement. Ann Intern Med 2014;160:330-338-8.

21 Kazerooni EA, Armstrong MR, Amorosa JK, et al. ACR CT Accreditation Program and the Lung Cancer Screening Program Designation. J Am Coll Radiol 2016;13:R30-R34.

22 Horeweg N, van Rosmalen J, Heuvelmans MA, et al. Lung cancer probability in patients with CT-detected pulmonary nodules: a prespecified analysis of data from the NELSON trial of low-dose CT screening. Lancet Oncol 2014;15:1332-41.

23 Hansell DM, Bankier AA, MacMahon H, et al. Fleischner Society: glossary of terms for thoracic imaging. Radiology 2008:246:697-722.

24 Field JK, Duffy SW, Baldwin DR, et al. UK Lung Cancer RCT Pilot Screening Trial: baseline findings from the screening arm provide evidence for the potential implementation of lung cancer screening. Thorax 2016;71:161-70.

25 Revel MP, Bissery A, Bienvenu M, et al. Are two-dimensional CT measurements of small noncalcified pulmonary nodules reliable? Radiology 2004;231:453-8.

26 Gietema HA, Schaefer-Prokop CM, Mali WP, et al. Pulmonary nodules: Interscan variability of semiautomated volume measurements with multisection CT-- influence of inspiration level, nodule size, and segmentation performance. Radiology 2007:245:888-94.

27 Swensen SJ, Silverstein MD, Ilstrup DM, et al. The probability of malignancy in solitary pulmonary nodules. Application to small radiologically indeterminate nodules. Arch Intern Med 1997;157:849-55.

28 Gould MK, Ananth L, Barnett PG; Veterans Affairs SNAP Cooperative Study Group. A clinical model to estimate the pretest probability of lung cancer in patients with solitary pulmonary nodules. Chest 2007;131:383-8.

29 Tammemagi MC, Freedman MT, Pinsky PF, et al. Prediction of true positive lung cancers in individuals with abnormal suspicious chest radiographs: a prostate, lung, colorectal, and ovarian cancer screening trial study. J Thorac Oncol 2009;4:710-21.

30 McWilliams A, Tammemagi MC, Mayo JR, et al. Probability of cancer in pulmonary nodules detected on first screening CT. N Engl J Med 2013;369:910-9.

31 Herder GJ, van Tinteren H, Golding RP, et al. Clinical prediction model to characterize pulmonary nodules: validation and added value of $18 \mathrm{~F}$-fluorodeoxyglucose positron emission tomography. Chest 2005;128:2490-6.

32 Walter JE, Heuvelmans MA, de Jong PA, et al. Occurrence and lung cancer probability of new solid nodules at incidence screening with low-dose CT: analysis of data from the randomised, controlled NELSON trial. Lancet Oncol 2016;17:907-16.

33 Cancer Research UK. Could a smartphone app help doctors diagnose lung cancer? 2016. http://scienceblog.cancerresearchuk.org/2016/12/06/could-a-smartphone-apphelp-doctors-diagnose-lung-cancer/ 